\title{
Caractérisation hydrochimique des aquifères fissurés de la région de San- Pedro (Sud-Ouest de la Côte d'Ivoire)
}

\author{
Théophile LASM $^{1 *}$, Omer DE LASME ${ }^{1}$, Marie-Solange Yéï OGA ${ }^{1}$, Marc YOUAN TA ${ }^{1,2}$, \\ Derving BAKA ${ }^{1}$, Fernand KOUAME ${ }^{1,2}$ et Théodore Koffi YAO ${ }^{1}$ \\ ${ }^{1}$ Laboratoire des Sciences et Techniques de l'Eau et du Génie de l'Environnement, U.F.R des Sciences de la \\ Terre et des Ressources Minières, Université de Cocody, 22 B.P. 582 Abidjan 22, Côte d'Ivoire; téléphone : \\ +22522483802 / +22522483803; télécopieur : + 22522445270 \\ ${ }^{2}$ Centre Universitaire de Recherche et d'Application en Télédétection (CURAT), U.F.R des Sciences de la \\ Terre et des Ressources Minières, Université de Cocody, 22 B.P. 801 Abidjan 22, Côte d'Ivoire; téléphone : \\ + 22522445270 ; télécopieur : + 22522445270 \\ *Auteur correspondant,E-mail : theophile.lasm@univ-cocody.ci,theophile_lasm@yahoo.fr
}

\section{RESUME}

La région de San-Pedro est située dans le Sud-Ouest de la Côte d'Ivoire. Son substratum est constitué de roches cristallines et métamorphiques fracturées. L'objectif de la présente étude est de mieux connaître les propriétés hydrochimiques et les mécanismes d'acquisition de la minéralisation des eaux souterraines de la région de San-Pedro. La base de données comporte les données des analyses physico-chimiques des eaux souterraines des aquifères fissurés et des fiches techniques des forages. L'approche hydrochimique et l'Analyse en Composantes Principales Normées ont été utilisées pour le traitement de ces données. Les résultats de cette étude montrent que les eaux souterraines de San-Pedro sont caractérisées par une faible minéralisation. Elles se subdivisent en deux principaux hydrofaciès : les eaux bicarbonatées et les eaux chlorurées. D'après le système calco-carbonique, on distingue trois familles d'eau dans la zone d'étude en fonction des indices de saturation vis-à-vis des carbonates : les eaux à circulation très lente, les eaux à circulation lente et les eaux à circulation rapide. L'ACPN a permis de mettre en évidence trois phénomènes principaux gouvernant la minéralisation des eaux souterraines : le temps de séjour de l'eau dans la roche aquifère, les apports superficiels et l'hydrolyse acide. Les eaux souterraines de la région de San-Pedro sont potables pour la consommation des populations humaines d'une manière générale. Ces résultats concourent à une meilleure connaissance des propriétés hydrochimiques des ressources en eaux souterraines du socle cristallin de Côte d'Ivoire.

(C) 2011 International Formulae Group. All rights reserved.

Mots clés : minéralisation, hydrofaciès, indices de saturation, eaux souterraines, âge relatif des eaux.

\section{INTRODUCTION}

Les ressources en eaux souterraines occupent une place de choix dans la politique d'approvisionnement en eau potable des populations rurales et urbaines en Côte d'Ivoire. Il apparaît donc nécessaire de mieux maîtriser ces ressources en eaux. Les aquifères fissurés sont de plus en plus exploités du fait de leur vaste répartition géographique $(97,5 \%$ $\mathrm{du}$ pays) et de leur importance hydrogéologique. Les qualités eaux de ces aquifères sont excellentes comparativement à 
celles des aquifères des altérites (Lasm, 2000). En effet, les couches des altérites qui surmontent les aquifères de fissures jouent deux rôles fondamentaux dans la protection des eaux à savoir (Lasm, 2000) : une filtration lente des micro-organismes et un effet tampon que le milieu fissuré ne peut assumer.

De nombreuses études (Lasm, 2000 ; Lasm et Razack, 2001 ; Jourda, 2005) ont été entreprises en Côte d'Ivoire en vue d'une meilleure connaissance des aquifères discontinus. Les résultats obtenus par ces auteurs sont fort encourageants et ont contribué à une meilleure connaissance de la géométrie de ces réservoirs. D'autres études sont en cours d'exécution afin de couvrir l'ensemble du pays.

En dépit des progrès considérables réalisés sur le continent africain en général et en Côte d'Ivoire en particulier dans le domaine de la recherche pour la mobilisation des ressources en eau potable, des efforts restent encore à faire. Dans un souci d'approvisionnement en eau potable, les autorités ivoiriennes avec l'appui de ses partenaires nationaux et internationaux ont réalisé plusieurs milliers d'ouvrages à travers les programmes d'Hydraulique Villageoise (HV) et d'Hydraulique Villageoise Amélioré (HVA). Dans la région de San-Pedro, de nombreux projets ont été réalisés parmi lesquels B.A.D.-1 (Banque Africaine de Développement) (1996-1997) et B.O.A.D.-3 (Banque Ouest Africaine de Développement) (1998-1999). Présentement, la région est dotée de plusieurs centaines de forages en exploitation. Ces forages captent essentiellement les eaux des aquifères de fissures et ont des profondeurs qui ne dépassent guère $90 \mathrm{~m}$. Les forages étaient implantés généralement sous des considérations géomorphologiques, ce qui avait pour conséquence l'obtention de faibles débits et un taux d'échec élevé. La combinaison des méthodes de géophysique et de photo-interprétations a donné des résultats très probants. Elle a permis non seulement de réduire le taux d'échec enregistré dans cette région mais aussi d'améliorer le débit des forages. En Côte d'Ivoire, on utilise de plus en plus les techniques de télédétection (imageries satellitaires et radars) pour la prospection hydrogéologique et l'implantation des forages. Le développement socio-économique de la région de San-Pedro a entrainé une augmentation des besoins en eaux. Le taux de couverture de ces besoins était de $43 \%$ en 2002, ce taux varie d'une région à une autre du pays en fonction du développement de celle-ci. Pour satisfaire aux différents besoins des populations de cette région, plusieurs projets de réalisation de points d'eau sont en cours d'exécution : i) projet 78 forages, piloté par l'ONG Solidarités San-Pedro avec l'appui des partenaires Européens, ii) projet FDPCC (Fonds de Développement et de Promotion des activités des Producteurs Café et de Cacao) ayant pour objectif la réalisation de 2000 forages, iii) projet PNUD avec pour objectif la réalisation de 25 forages dans le Bas Sassandra, iv) etc.

Dans la région Sud-ouest, en dehors des études pétrographiques et tectoniques (Kouamelan et al., 1997), les études hydrogéologiques sont quasi-inexistantes. Le contexte hydrogéologique est donc très peu connu, ce qui constitue un handicap pour une meilleure exploitation et gestion des ressources en eaux souterraines. En effet, la modélisation des écoulements souterrains nécessite une meilleure connaissance des propriétés hydrogéologiques et hydrochimiques des aquifères fissurés. C'est donc dans le souci d'apporter une contribution à la connaissance hydrogéologique des aquifères du Sud-Ouest de la Côte d'Ivoire que plusieurs travaux ont été entrepris. L'un des volets de ces travaux concerne la caractérisation hydrochimique des aquifères fissurés. Cette étude a pour objectif de mieux connaître les propriétés hydrochimiques et les mécanismes d'acquisition de la minéralisation 
des eaux souterraines de la région de SanPedro. Elle intéresse une région où les différents événements tectoniques ont abouti à une fracturation très développée. L'étude de la fracturation ne sera pas abordée ici, car elle sort des limites de ce travail.

\section{Contextes géographique et géologique de la région de San-Pedro}

Située dans le Sud-Ouest de la Côte $\mathrm{d}^{\prime}$ Ivoire, entre les latitudes $4^{\circ} 15^{\prime} \mathrm{N}$ et $5^{\circ} 30^{\prime}$ $\mathrm{N}$, et les longitudes $6^{\circ} 15 \mathrm{~W}$ et $7^{\circ} 20 \mathrm{~W}$, la région de San-Pedro couvre une superficie d'environ $6912 \mathrm{~km}^{2}$.

La population de cette région a été estimée en 2000, à environ 244600 habitants (Géohive, 2008). Selon les estimations du Ministère des Infrastructures Economiques, cette population avoisinerait 355800 habitants à l'horizon 2010.

La région est composée de deux entités géomorphologiques distinctes : une pédiplaine caractérisée par une forêt sempervirente, située à l'Est de la zone d'étude, et une frange littorale rocheuse localisée au Sud-Ouest. Le régime climatique est de type équatorial de transition avec deux saisons de pluies et deux saisons sèches. La pluviométrie annuelle moyenne est comprise entre 1700 et 2000 mm (Géomines, 1982).

Le sous sol de la région de San-Pedro est essentiellement composé de gneiss, migmatites, granites, granodiorites, pegmatites, micaschistes, grauwackes, flyschs et de nombreux filons de quartz et de dolérites (Figure 1). D'un point de vue tectonique et structural, cette région se singularise par une poly déformation caractérisée par des mécanismes d'aplatissements et de cisaillements ductiles avec un métamorphisme de type rétrograde.

Deux déformations majeures ont affecté le Sud-Ouest de la Côte d'Ivoire et notamment la région de San-Pedro (Yacé, 2002) : i) une tectonique tangentielle caractérisée par le style plicatif et la mise en évidence des plis isoclinaux à plans axiaux sub-verticaux, ii) une tectonique cassante caractérisée par de nombreuses fractures de tailles variables. L'ensemble des déformations a abouti à la mise en place d'une fracturation développée à l'instar de l'ouest de la Côte d'Ivoire. L'étude de la fracturation ne sera pas abordée ici.

$\mathrm{Au}$ plan hydrogéologique, on $\mathrm{y}$ rencontre deux types d'aquifères: les aquifères des altérites et de fissures. Ces deux aquifères sont issus respectivement de l'altération et de la fracturation des roches dures cristallines et cristallophylliennes. Les aquifères des altérites et de fissures sont dans bien de cas superposés et sont étroitement liés par un phénomène de drainance (Lasm, 2000). Les aquifères de fissures drainent les aquifères des altérites qui leur sont superposés. Il peut arriver parfois que l'aquifère des altérites fasse défaut ou bien soit disposé latéralement, partiellement saturé ou pas, drainé ou drainant.

Les aquifères fissurés sont à l'abri de toute fluctuation saisonnière et d'éventuelles pollutions du fait de leur grande profondeur. Les aquifères fissurés offrent de bien meilleures garanties d'un point de vue sécuritaire que les aquifères des altérites. C'est la raison pour laquelle les aquifères fissurés constituent les aquifères les plus recherchés et exploités dans les programmes d'hydraulique villageoise et urbaine en Côte d'Ivoire.

\section{Données et méthodes \\ Données}

Les données hydrochimiques utilisées sont essentiellement les résultats des analyses physico-chimiques des échantillons d'eau prélevés à la fin de la saison des pluies, dans 45 forages de la région (Figure 1). Ces forages ont été retenus en raison de la qualité des résultats des analyses chimiques. Ces données ont été obtenues au cours des projets d'hydrauliques villageoises et urbaines exécutés par les sociétés FORACO-CI 
(Compagnie de forages en Côte d'Ivoire) et C.G.C.-CI (Compagnie Géologique Canadienne en Côte d'Ivoire) respectivement en 1996-1997 et en 1998-1999. Les analyses des échantillons d'eau ont été effectuées au Laboratoire National d'Essais de Qualité, de Métrologie et d'Analyses (LANEMA) à Abidjan (Côte d'Ivoire).

La température $\left(\mathrm{T}^{\circ}\right)$, la conductivité électrique (CE) et le $\mathrm{pH}$ ont été mesurés sur le terrain et des échantillons d'eau ont été recueillis pour le dosage des ions. Le $\mathrm{pH}$ et la conductivité ont été mesurés respectivement à l'aide d'un pH-mètre et d'un conductimètre numérique dont les incertitudes sont de $\pm 0,1$ et $\pm 0,1 \mu \mathrm{S} . \mathrm{cm}^{-1}$. La température a été mesurée à l'aide d'un thermomètre dont l'incertitude est de $\pm 0,1^{\circ} \mathrm{C}$.

Ces échantillons ont été conditionnés et stockés à l'abri de la poussière et de la lumière à une température de $4{ }^{\circ} \mathrm{C}$. Les éléments majeurs et autres ont été dosés au laboratoire par différentes techniques. Les ions $\mathrm{HCO}_{3}^{-}$, $\mathrm{Cl}^{-}, \mathrm{NO}_{3}{ }^{-}, \mathrm{SO}_{4}^{2-}, \mathrm{Ca}^{2+}, \mathrm{Mg}^{2+}, \mathrm{Na}^{+}, \mathrm{Fe}^{2+}$ et $\mathrm{Mn}^{2+}$ ont été dosés au laboratoires une quinzaine de jours au maximum après les prélèvements. Les ions $\mathrm{Cl}^{-}$et $\mathrm{HCO}_{3}{ }^{-}$ont été déterminés par titration par ajout de nitrate d'argent de molarité $0,016 \mathrm{~N}$ en présence de $5 \%$ de bichromate de potassium comme indicateur, les bicarbonates ont été mesurés à l'aide de $0,1 \mathrm{~N}$ d'acide chlorhydrique en utilisant le méthyle orange comme indicateur coloré. Les sulfates ont été dosés par néphélométrie, les nitrates et phosphates ont été dosés par colorimétrie. Tous les cations ont été dosés avec un spectrophotomètre à flamme d'absorption atomique de marque SHIMATSU. Les incertitudes des résultats d'analyses sont respectivement de $2 \%$ pour les cations et $1 \%$ pour les anions.

Le logiciel STATISTICA $^{\text {TM }} 6.0$ de STATSOFT a été utilisé pour l'Analyse en Composantes Principales Normées (ACPN) et le logiciel d'hydrochimie DIAGRAMME 4.00 pour les interprétations des données hydrochimiques.

\section{Méthodes}

Méthode de détermination des indices de saturation ISC et ISD dans les eaux souterraines

Les variables du système calcocarbonique qui permettent de déterminer le temps de séjour relatif des eaux souterraines sont la pression partielle de $\mathrm{CO}_{2}\left(\mathrm{pCO}_{2}\right)$, le $\mathrm{pH}$ d'équilibre $\left(\mathrm{pH}_{\mathrm{eq}}\right)$ et les indices de saturation de l'eau par rapport à la calcite (ISC) et à la dolomite (ISD).

Les indices ISC et ISD peuvent être calculés respectivement à l'aide des équations (1) et (2) :

$$
\begin{aligned}
& I S C=p H_{\text {réel }}-p H_{e q}=\Delta p H \quad \ldots \ldots . .(1) \\
& I S D=p k_{D}-2\left[p H-p k_{2}+\log \left(H C O_{3}^{-}\right)+\log \left(M g^{2+}\right)\right]
\end{aligned}
$$

$\mathrm{pH}_{\text {réel, }}$, et $\mathrm{pH}_{\mathrm{éq}}$, sont respectivement les $\mathrm{pH}$ mesuré et calculé. Le pH d'équilibre $\left(\mathrm{pH}_{\mathrm{eq}}\right)$ est égal au $\mathrm{pH}$ d'une eau en équilibre chimique avec un carbonate, pour une activité invariable du bicarbonate.

Pour plus de détails sur cette approche, nous renvoyons le lecteur à consulter la littérature car elle a été bien développée.

$$
\text { Lorsque } \Delta p H=p H_{\text {réel }}-p H_{e ́ q}=0 \text {, }
$$

l'eau est en équilibre. Dans ce cas, la solution ne peut subir d'évolution tant que les conditions de $\mathrm{pCO}_{2}$ et de température n'auront pas variées. Une telle eau est dite saturée visà-vis des carbonates considérés. Cela se traduit par un épuisement de $\mathrm{CO}_{2}$ initialement présent dans l'eau, une vitesse de circulation lente des eaux et un âge relativement avancé.

$\mathrm{Si} \Delta \mathrm{pH}>0$, les eaux sont dites sursaturées vis-à-vis de la calcite. Ces eaux peuvent être considérées «très anciennes », car seul un contact très prolongé entre l'eau et l'aquifère peut justifier la présence de précipité de carbonates dans les eaux souterraines. En effet, au contact du $\mathrm{CO}_{2}$ dissous et de l'acide carbonique $\mathrm{H}_{2} \mathrm{CO}_{3}$, les 
minéraux ferromagnésiens favorisent l'apparition des carbonates purement secondaires et susceptibles d'accroître les indices de saturation de l'eau en carbonates secondaires vers des valeurs nulles et positives. Les eaux caractérisées par des valeurs positives de $\Delta \mathrm{pH}$ sont des eaux non agressives qui circulent très lentement dans l'aquifère.

Lorsque $\Delta \mathrm{pH}<0$, les eaux sont soussaturées vis-à-vis des carbonates (calcite). Dans ce cas, les eaux sont généralement riches en $\mathrm{CO}_{2}$ dissous et très agressives avec une grande vitesse de circulation. Ces eaux peuvent être considérées «récentes» dans l'aquifère car elles sont en perpétuelle réalimentation par les eaux d'infiltration.

Le diagramme ISD $=\mathrm{f}($ ISC) permet de mettre en évidence : i) l'alignement des points représentatifs des eaux en fonction de leur proportion en carbonates; ii) le regroupement des échantillons en familles selon le temps de séjour des eaux; iii) la perméabilité des aquifères; et iv) la vitesse de circulation des eaux.

Méthode de l'analyse en Composantes Principales Normées (ACPN)

L'Analyse en Composante Principale Normée est un outil puissant utilisé pour mettre en évidence les ressemblances chimiques entre les différentes eaux et de comprendre les différents mécanismes à l'origine de la minéralisation des eaux souterraines. L'ACPN remplace un tableau de nombres difficiles à lire par une représentation plus facile à lire sous forme de nuage de points.

Ce type de représentation d'un échantillon permet de visualiser tous les individus et d'interpréter leur disposition afin de déterminer les principaux facteurs responsables de la structure observée aussi bien par rapport aux variables que par rapport aux unités statistiques (u.s.). L'usage de l'ACPN permet d'éliminer l'effet de taille et l'hétérogénéité que l'on peut enregistrer au niveau des données.

Dans l'espace des variables, les coordonnées de chaque variable correspondent aux saturations. Une variable contribue d'autant plus à la détermination d'un facteur qu'elle est éloignée du centre de gravité. Dans l'espace des u.s. on recherche la contribution de chaque u.s. à l'inertie du nuage, puis leurs composantes principales et enfin la contribution de chaque u.s. à la détermination des facteurs. Nous renvoyons le lecteur pour plus de détails à consulter les travaux de (Lasm et al., 2008b). L'Analyse en Composantes Principales Normées (ACPN) a été réalisée avec 9 principales variables. Les variables étudiées sont : $\mathrm{Ca}^{2+}, \mathrm{Mg}^{2+}, \mathrm{Na}^{+}, \mathrm{Fe}^{2+}$, $\mathrm{HCO}_{3}^{-}, \mathrm{Cl}^{-}, \mathrm{NO}_{3}^{-}, \mathrm{MTD}$ (matières totales dissoutes) et $\mathrm{pCO}_{2}$ (pression partielle de $\mathrm{CO}_{2}$ ). Ces différents paramètres physico-chimiques ont été mesurés ou calculés au niveau de 45 échantillons d'eau de forages (u.s.).

\section{RESULTATS}

\section{Analyse des paramètres physiques}

Les valeurs des paramètres physiques des eaux souterraines sont consignées dans le Tableau 1. L'analyse du Tableau 1 montre que les eaux souterraines de San-Pedro ont une température moyenne de $25,6{ }^{\circ} \mathrm{C}$ dans des forages dont la profondeur n'excède pas $90 \mathrm{~m}$. Ces eaux sont plus ou moins neutres $(6,5<\mathrm{pH}$ <7) à 33\%, acides $(\mathrm{pH}<6,5)$ à $29 \%$ et basiques $(\mathrm{pH}>7)$ à $38 \%$. La gamme de variation des valeurs du $\mathrm{pH}$ de ces eaux permet de les classer dans le domaine des eaux à alcalinité bicarbonatée $(4,5<\mathrm{pH}<$ 9,5).

Les valeurs extrêmes de la conductivité électrique ont été obtenues dans les eaux de forage de Golykouassikro (31 $\left.\mu \mathrm{S} . \mathrm{cm}^{-1}\right)$ et Kandakro $\left(667 \mu \mathrm{S} . \mathrm{cm}^{-1}\right)$. Celles de la pression partielle du gaz carbonique $\left(\mathrm{pCO}_{2}\right)$ dissoute dans les eaux sont 0,00 atm et 0,07 atm. Les valeurs de $\mathrm{CO}_{2}$ déduites varient entre 0,05 mg.L ${ }^{-1}$ (Ech. 31 : Belem F1) et 101,98 mg.L ${ }^{-1}$ 
(Ech. 40 : Nematoulaye) avec une moyenne de $38,18 \mathrm{mg} \cdot \mathrm{L}^{-1}$.

La quantité de matières totales dissoutes (MTD) des eaux souterraines oscille entre 62,85 (Ech.32: Belem 2) et 741,74 mg.. ${ }^{-1}$ (Ech. 14 : Amadoukro) avec une moyenne et un écart-type respectivement de 292,08 et 149,82 mg. $\mathrm{L}^{-1}$. Ce résultat montre que les eaux sont douces (MTD $<1000 \mathrm{mg} . \mathrm{L}^{-1}$ ) et leur taux de minéralisation n'est pas identique en tout point de la zone d'étude.

La dureté totale des eaux ou encore le Titre Hydrotimétrique Total (THT), varie de 2 (Ech. 29: Djihimbo) à $50{ }^{\circ} \mathrm{F}$ (Ech. 14 : Amadoukro) avec une moyenne de $11,6{ }^{\circ} \mathrm{F}$. La dureté des eaux de San-Pedro se répartie comme suit: $51 \%$ des eaux ont une dureté faible (THT<10 $\left.{ }^{\circ} \mathrm{F}\right), 44 \%$ sont caractérisées par une dureté normale $\left(10<\mathrm{THT}<30^{\circ} \mathrm{F}\right)$ et $5 \%$ présentent une dureté indésirable (THT $>30^{\circ} \mathrm{F}$ ).

\section{Analyse des éléments majeurs}

La teneur du sodium oscille entre 1,86 (Ech. 10 : Daro) et 147,70 mg.L ${ }^{-1}$ (Ech. 31 : Belem), avec une moyenne de $22,64 \mathrm{mg} . \mathrm{L}^{-1}$ et une médiane de 16,70 mg. $\mathrm{L}^{-1}$. Les valeurs ne sont pas homogènes. En effet, la majorité des échantillons $(98 \%)$ oscillent entre 1,86 et $57,13 \mathrm{mg} \cdot \mathrm{L}^{-1}$.

Celle du potassium est comprise entre 0,90 (Ech. 29: Djihimbo) et 78,30 mg.L $\mathrm{L}^{-1}$ (Ech. 5: Zilekro) avec une moyenne et une médiane respectivement de $21,84 \mathrm{mg} . \mathrm{L}^{-1}$ et $8,50 \mathrm{mg} \cdot \mathrm{L}^{-1}$. Les alcalins sont faiblement présents dans les eaux souterraines avec $15 \%$ des MTD.

Le calcium et le magnésium ont des teneurs qui évoluent respectivement de 2,70 (Ech. 1 : Dagadji ) à 142,30 mg.L ${ }^{-1}$ (Ech. 14 : Amadoukro) et de 1,80 (Ech. 6 : Adamakro) à 34,35 mg.L $\mathrm{L}^{-1}$ (Ech. 14 : Amadoukro) avec une moyenne de 28,52 mg. $\mathrm{L}^{-1}$ et de $10,80 \mathrm{mg} . \mathrm{L}^{-1}$. Les valeurs médianes de ces deux éléments sont respectivement égales à $19,30 \mathrm{mg} . \mathrm{L}^{-1}$ et 9,00 mg. $\mathrm{L}^{-1}$. Les eaux de la région sont légèrement plus calciques que magnésiennes car le calcium représente $10 \%$ des matières totales dissoutes tandis que le magnésium n'en représente que $4 \%$. La teneur en fer est comprise dans l'intervalle de 0,05 (Ech. 24: Taboké) à 3,70 mg.L L $^{-1}$ (Ech. 9 : Yaokro), avec une moyenne de $0,72 \mathrm{mg} . \mathrm{L}^{-1}$ et une médiane de $0,6 \mathrm{mg} \cdot \mathrm{L}^{-1}$. Le fer a une proportion de $0,2 \%$ dans l'ensemble des ions de la région.

Le bicarbonate est l'anion prédominant dans les eaux souterraines étudiées car sa teneur représente environ $53 \%$ de la minéralisation totale. Sa concentration dans les eaux prélevées varie de 1,68 pour le forage de Belem 2 (Ech. 32) à 384,30 mg.L L $^{-1}$ pour le forage de SCAF (Ech. 2), avec une moyenne de $156,02 \mathrm{mg} . \mathrm{L}^{-1}$ et une médiane de 124 mg. $\mathrm{L}^{-1}$. Le chlore a une teneur comprise entre 5,32 (Ech. 33 : Djapadji 1) et $67,10 \mathrm{mg} . \mathrm{L}^{-1}$ (Ech. 5 : Zilekro) avec une moyenne de 14,84 mg. $\mathrm{L}^{-1}$ et une médiane de $11 \mathrm{mg} . \mathrm{L}^{-1}$. Le chlore représente $5 \%$ de la minéralisation totale. Les nitrates sont faiblement présents dans les eaux de forages étudiées; leurs teneurs oscillent entre 0,40 (Ech. 5 : Zilekro) et 52,88 mg. $\mathrm{L}^{-1}$ (Ech. 24 : Taboké) soit une moyenne de 5,73 mg. $\mathrm{L}^{-1}$ et une médiane de $2,58 \mathrm{mg} . \mathrm{L}^{-1}$. Les nitrates $\left(\mathrm{NO}_{3}{ }^{-}\right)$et nitrites représentent $2 \%$ de MTD. Les sulfates sont plus présents dans ces eaux souterraines que les nitrates avec une teneur moyenne de $30,98 \mathrm{mg} . \mathrm{L}^{-1}$ et une médiane de $12,8 \mathrm{mg} . \mathrm{L}^{-1}$. Les sulfates ont une teneur comprise entre 0,45 (Ech. 12: Jeanokro) et 229,40 mg.L ${ }^{-1}$ (Ech. 14 : Amadoukro) et représente environ $11 \%$ des MTD.

\section{Faciès hydrochimiques}

La classification des eaux souterraines de San-Pedro d'après le diagramme de Piper met en évidence quatre principaux types de faciès chimiques (Figure 2): i) eaux bicarbonatées calciques et magnésiennes; ii) eaux bicarbonatées sodiques et potassiques; iii) eaux chlorurées sulfatées calciques et 
magnésiennes; iv) et eaux chlorurées sodiques et potassiques.

Le faciès bicarbonaté calcique et magnésien caractérise plus de la moitié des eaux de la région du Sud-Ouest de la Côte d'Ivoire, soit $60 \%$ des échantillons étudiés. Les anions dominants dans ces eaux sont les bicarbonates $\left(\mathrm{HCO}_{3}{ }^{-}\right)$tandis que le calcium et le magnésium $\left(\mathrm{Ca}^{2+}>\mathrm{Mg}^{2+}\right)$ sont les cations prédominants. Ces eaux se rencontrent majoritairement dans les aquifères fissurés des migmatites et des micaschistes.

Le faciès bicarbonaté sodique et potassique représente environ $20 \%$ des échantillons d'eau étudiés. $\mathrm{Ce}$ faciès hydrochimique est suffisamment présent dans les aquifères de gneiss et de micaschistes. L'ordre de prédominance des ions de ces eaux s'établit comme suit : $\mathrm{Cl}^{-}>\mathrm{NO}_{3}{ }^{-}$et $\mathrm{Na}^{+}>\mathrm{K}^{+}$.

Le faciès chloruré sulfaté calcique et magnésien représente environ $11 \%$ des eaux souterraines. L'ordre de prédominance des ions dans ces eaux est établi de la façon suivante : $\mathrm{Cl}^{-}>\mathrm{SO}_{4}{ }^{2-}$ pour les anions et $\mathrm{Ca}^{2+}>$ $\mathrm{Mg}^{2+}$ pour les cations. De fortes teneurs en sulfates sont rencontrées dans différentes localités et dans différents types de roches: Amadoukro (Ech. 14), à Taboké (Ech. 24) et à Henrikro (Ech. 30) respectivement dans les granodiorites, les migmatites et les gneiss.

Le faciès chloruré sodique et potassique est le moins présent dans la région de SanPedro, car il regroupe seulement $9 \%$ des eaux de forages prélevées dans des formations de gneiss et de micaschiste. Concernant l'abondance des ions dans ce type de faciès d'eaux souterraines, l'anion $\mathrm{Cl}^{-}$l'emporte sur $\mathrm{NO}_{3}{ }^{-}$et le cation $\mathrm{Na}^{+}$prédomine $\mathrm{K}^{+}$.

\section{Circulation des eaux souterraines de la région de San-Pedro}

Sur le graphe ISD = f(ISC) (Figure 3), les points représentants les échantillons d'eau s'alignent suivant une droite sur laquelle il est possible d'ajuster une droite de régression d'équation :
$I S D=1,89 \times I S C-0,67$

avec ISD et ISC respectivement les indices de saturation vis-à-vis de la dolomite et de la calcite.

Le coefficient de détermination $\left(\mathrm{R}^{2}=\right.$ 0,9794) indique que le test statistique est satisfaisant.

La majorité des eaux de forages San-Pedro sont sous-saturées vis-à vis de la calcite et de la dolomite en dehors de six échantillons (Tableau 1). La répartition des points dans le diagramme permet de classer les eaux souterraines en trois catégories différentes en fonction de leur vitesse de circulation. Il s'agit des familles d'eaux à circulation très lentes, lentes et rapides (Figure 3).

Les eaux très lentes (Famille 1) représentent $13 \%$ des échantillons. Elles présentent un indice de saturation vis-à vis de la calcite strictement positif (ISC > 0) et une teneur moyenne en $\mathrm{CO}_{2}$ dissous égale à 19,23 mg. $\mathrm{L}^{-1}$. Ces valeurs traduisent une très faible agressivité des eaux vis-à-vis des roches encaissantes; et par conséquent une circulation très lente dans les aquifères qui les contiennent. Ces eaux souterraines sont celles des localités d'Amadoukro $\left(\mathrm{CO}_{2}\right.$ dis $\left.=33,34\right)$, Bledje $\left(\mathrm{CO}_{2}\right.$ dis $\left.=21,34 \mathrm{mg} . \mathrm{L}^{-1}\right)$, Lamenée $\left(\mathrm{CO}_{2}\right.$ dis $\left.=10,06 \mathrm{mg} \cdot \mathrm{L}^{-1}\right), \quad$ Benjaminkro $\left(\mathrm{CO}_{2}\right.$ dis $=4,23$ mg. $\left.\mathrm{L}^{-1}\right), \quad$ Kouassidrokro $\left(\mathrm{CO}_{2}\right.$ dis $\left.=18,69 \mathrm{mg} \cdot \mathrm{L}^{-1}\right)$ et Dozo-Djilé $\left(\mathrm{CO}_{2}\right.$ dis $\left.=27,17 \mathrm{mg} \cdot \mathrm{L}^{-1}\right)$. Ces eaux sont caractérisées par une quantité importante de MTD (431 à $742 \mathrm{mg} . \mathrm{L}^{-1}$ ) soit une valeur moyenne de 536,18 mg.. $\mathrm{L}^{-1}$. En effet, la minéralisation de l'eau souterraine se fait à la faveur de l'interaction eau-roche.

Les eaux de la Famille 2 représentent $56 \%$ des eaux étudiées, avec un indice de saturation en calcite compris entre -1,5 < ISC $<0$. La teneur en $\mathrm{CO}_{2}$ dissous s'échelonne entre 0,05 et $56,65 \mathrm{mg} . \mathrm{L}^{-1}$ avec une teneur moyenne de $32,54 \mathrm{mg} . \mathrm{L}^{-1}$. Ces eaux sont moins agressives avec une vitesse de circulation lente. Les aquifères de ces eaux 
sont caractérisés par une perméabilité probablement moyenne.

Les eaux de la Famille 3 représentent $31 \%$ des eaux souterraines. Ces eaux à faible MTD (62,85 à 316,77 mg.. $\left.{ }^{-1}\right)$, avec une valeur moyenne de $174,93 \mathrm{mg} . \mathrm{L}^{-1}$ sont caractérisées par des indices de saturation vis-à-vis des carbonates secondaires les plus négatifs (ISC $<-1,5$ et ISD $<-4)$. La teneur moyenne en $\mathrm{CO}_{2}$ dissous est la plus élevée, soit 49,22 $\mathrm{mg} . \mathrm{L}^{-1}$. Ces eaux sont très agressives en raison de leur forte teneur en $\mathrm{CO}_{2}$ dissous et la très faible saturation de ces eaux vis-à-vis des carbonates secondaires. Les eaux ont une circulation rapide dans des aquifères certainement bien fissurés avec de bonnes propriétés géométriques et/ou les altérites suffisamment perméables.

\section{Origines et processus de minéralisation des eaux souterraines}

La matrice de corrélation met en évidence les différentes corrélations existantes entre les paramètres étudiés. Les corrélations les plus significatives dans cette matrice (Tableau 2) sont celles existant entre les alcalino-terreux $\mathrm{Ca}^{2+}$ et $\mathrm{Mg}^{2+}(0,77)$, MTD et $\mathrm{Ca}^{2+}(0,85)$, MTD et $\mathrm{Mg}^{2+}(0,78)$, MTD et $\mathrm{HCO}_{3}^{-}(0,83)$. Il ressort de ces valeurs de corrélation que les couples de variables ont une origine commune, ils évoluent dans le même sens et sont produits par des phénomènes naturels identiques.

Les trois premiers axes factoriels expriment $71,2 \%$ de la variance totale dont $38,7 \%$ pour le premier, $20 \%$ pour le second et $12,4 \%$ pour le troisième (Figures 4 et 5 ). Cette variance est suffisante pour fournir des renseignements sur les interactions hydrogéochimiques qui se déroulent dans les aquifères de la région. Le pourcentage cumulé de la variance exprimée par les deux premiers facteurs $(58,8 \%)$ et les regroupements des variables dans le plan F1-F2 rend compte de façon satisfaisante de leur structure. Cependant, certains aspects de cette structure seront peut être mal identifiés puisqu'il reste encore 41,2\% de variance à exprimer. C'est la raison pour laquelle l'analyse se poursuit jusqu'au facteur 3 (Figures 6 et 7). C'est dans le plan des variables que chacun des facteurs sera identifié.

\section{Analyse dans l'espace des variables et des unités statistiques}

L'analyse du cercle de communauté du plan factoriel F1-F2 (Figure 4) permet de dégager deux principaux regroupements des variables. Le premier regroupement est situé dans la partie positive de l'axe F1 et concerne MTD, $\mathrm{Ca}^{2+}, \mathrm{Mg}^{2+}$ et $\mathrm{HCO}_{3}^{-}$. Le deuxième regroupement est situé à l'extrémité positive de l'axe $\mathrm{F} 2$ et comprend le $\mathrm{Cl}^{-}$et $\mathrm{Na}^{+}$.

Le regroupement de MTD avec deux cations majeurs et un anion majeur suivant l'axe F1 suggère leur origine commune et un même mécanisme de minéralisation. En effet, les ions $\mathrm{Mg}^{2+}, \mathrm{Ca}^{2+}$ et $\mathrm{HCO}_{3}{ }^{-}$apparaissent dans l'eau après un contact prolongé des eaux souterraines avec les roches encaissantes. Le mécanisme d'enrichissement de ces eaux en alcalino-terreux et bicarbonates est la conséquence d'une hydrolyse acide des roches contenant des minéraux silicatés. En conséquence, le facteur 1 exprime le phénomène de la minéralisation lié au temps de séjour des eaux souterraines dans l'aquifère.

Dans le second regroupement, la proximité du $\mathrm{Cl}^{-}$et $\mathrm{du} \mathrm{Na}^{+}$suggère la provenance d'une source commune de ces ions et un mécanisme de minéralisation identique. Le phénomène naturel favorisant la salinité des eaux souterraines est certainement l'influence marine. Cette interprétation est en accord avec la proximité de la mer et l'importante pluviométrie (soit environ 1700 $\mathrm{mm}$ par an) de la région de San-Pedro. En effet, la région de San-Pedro étant située sur le littoral, les masses de vapeur précipitantes d'origine essentiellement océaniques sont riches en $\mathrm{Na}^{+}$et $\mathrm{Cl}^{-}$provenant de l'évaporation de l'eau de mer. Les eaux de pluies riches en $\mathrm{Na}^{+}$et $\mathrm{Cl}^{-}$vont atteindre les aquifères souterrains par infiltration. Le facteur 2 exprime donc l'influence de la mer par l'infiltration des eaux de pluies. 


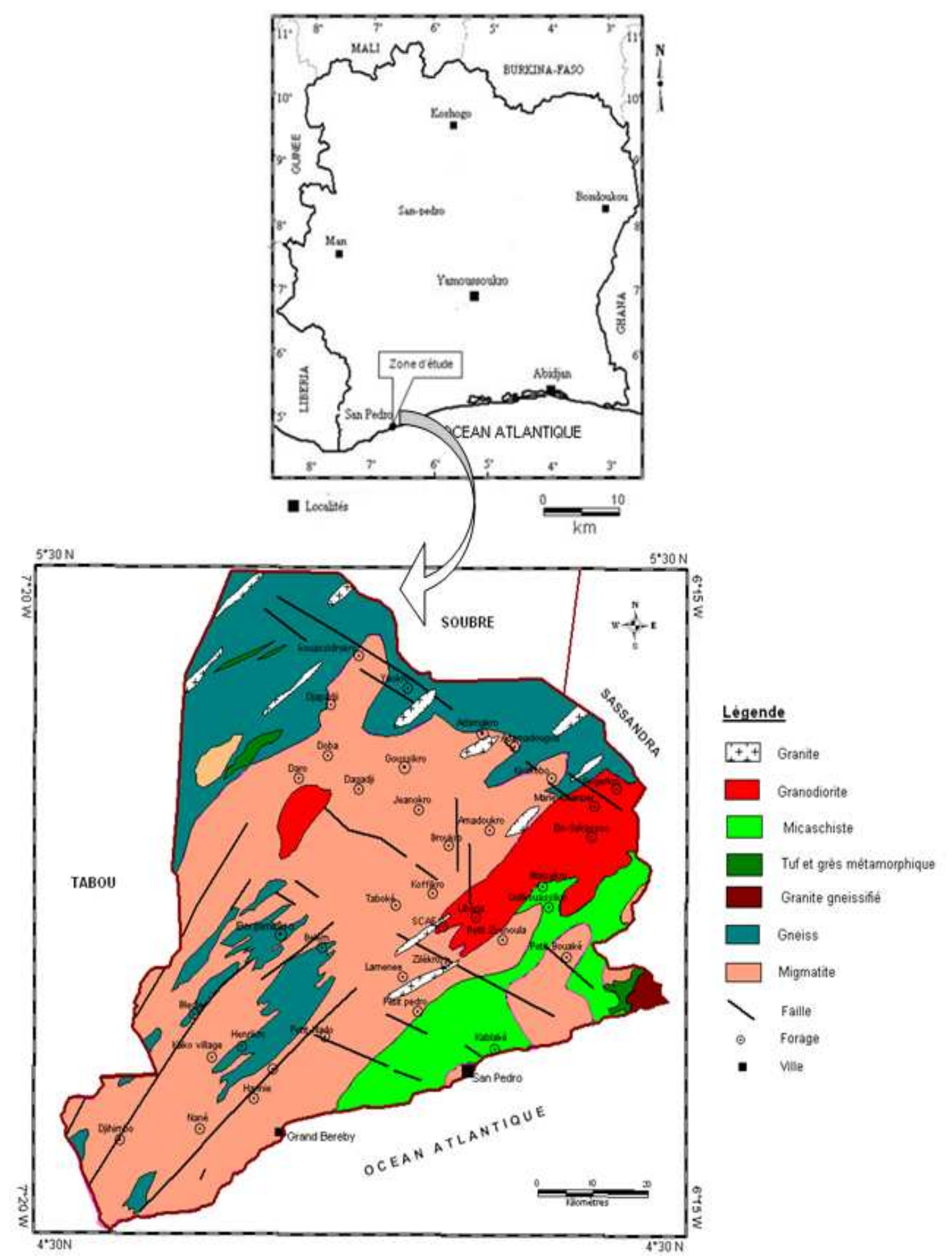

Figure 1 : Présentation de la zone d'étude et carte géologique de la région de San-Pedro. 
Eau bicarbonatée calcique et magnésienne

$\int$ Eau bicarbonatée sodique et potassique $\checkmark \begin{aligned} & \text { Eau chlonurée sul fatée calcique et } \\ & \text { magnésienne }\end{aligned}$

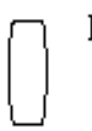

Eau chlonurée sodique et potassique

-

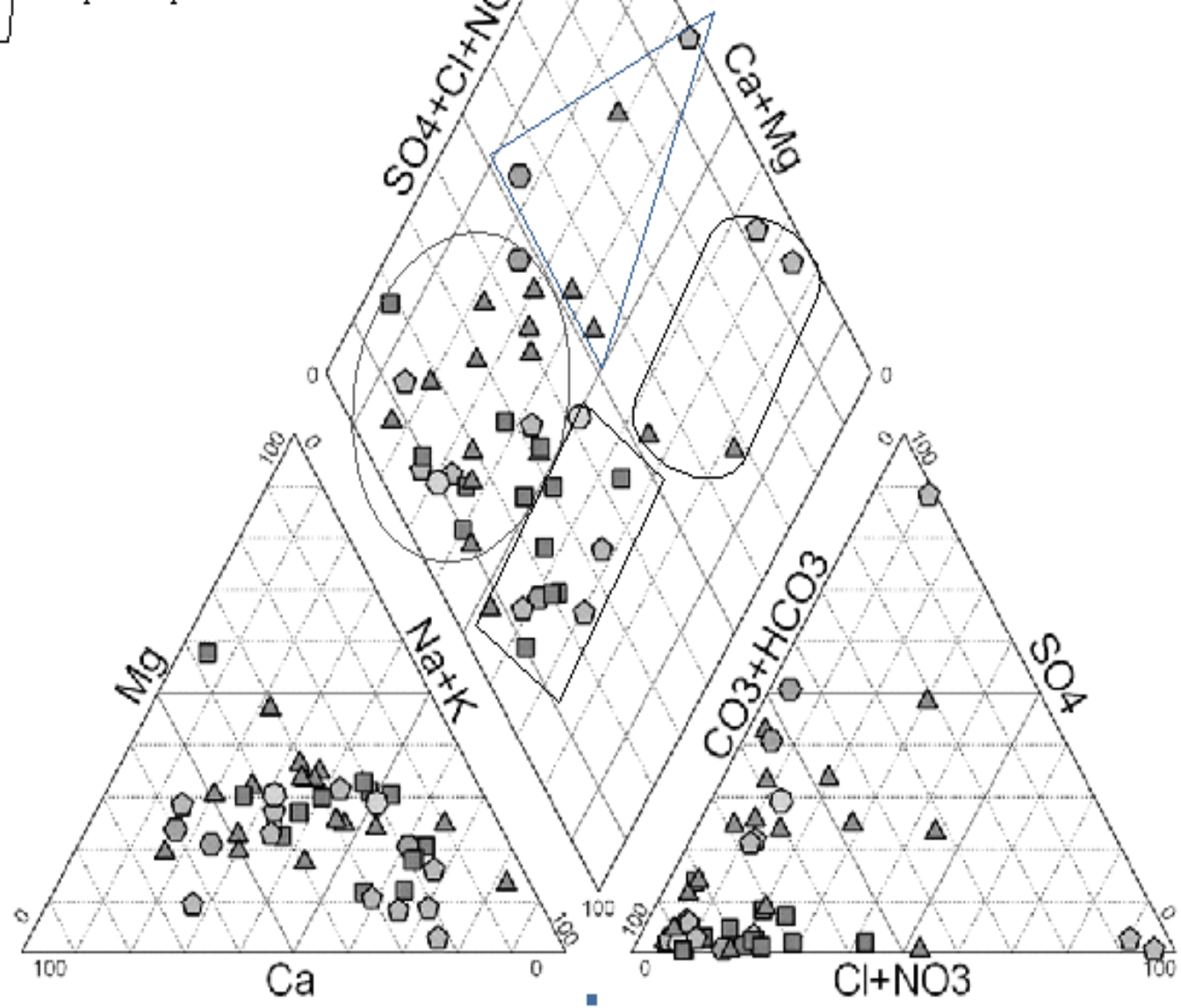

Figure 2 : Classification des eaux souterraines de San-Pedro dans le diagramme de Piper. 


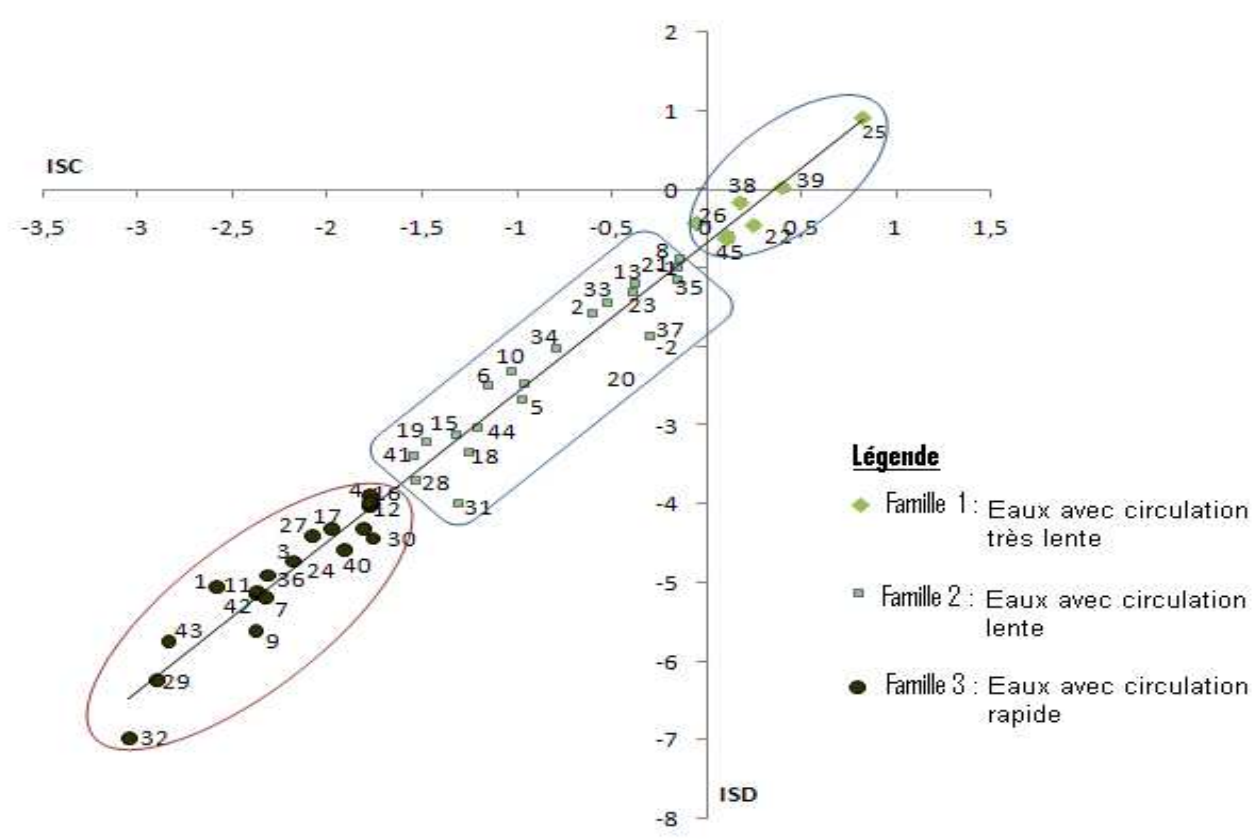

Figure 3 : Diagramme ISD/ISC des eaux souterraines de la région de San-Pedro.

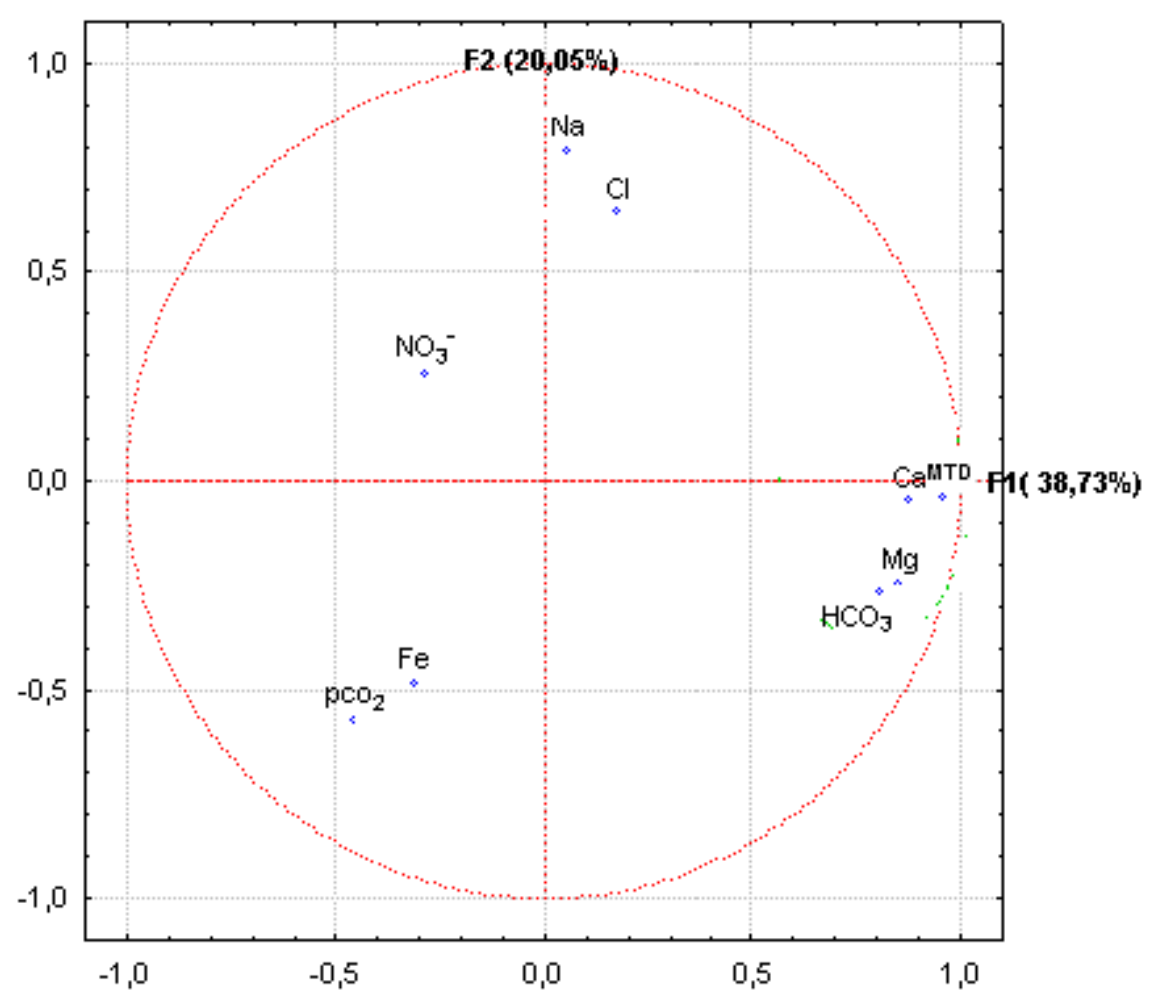

Figure 4 : Projection des variables dans l'espace factoriel F1-F2. 


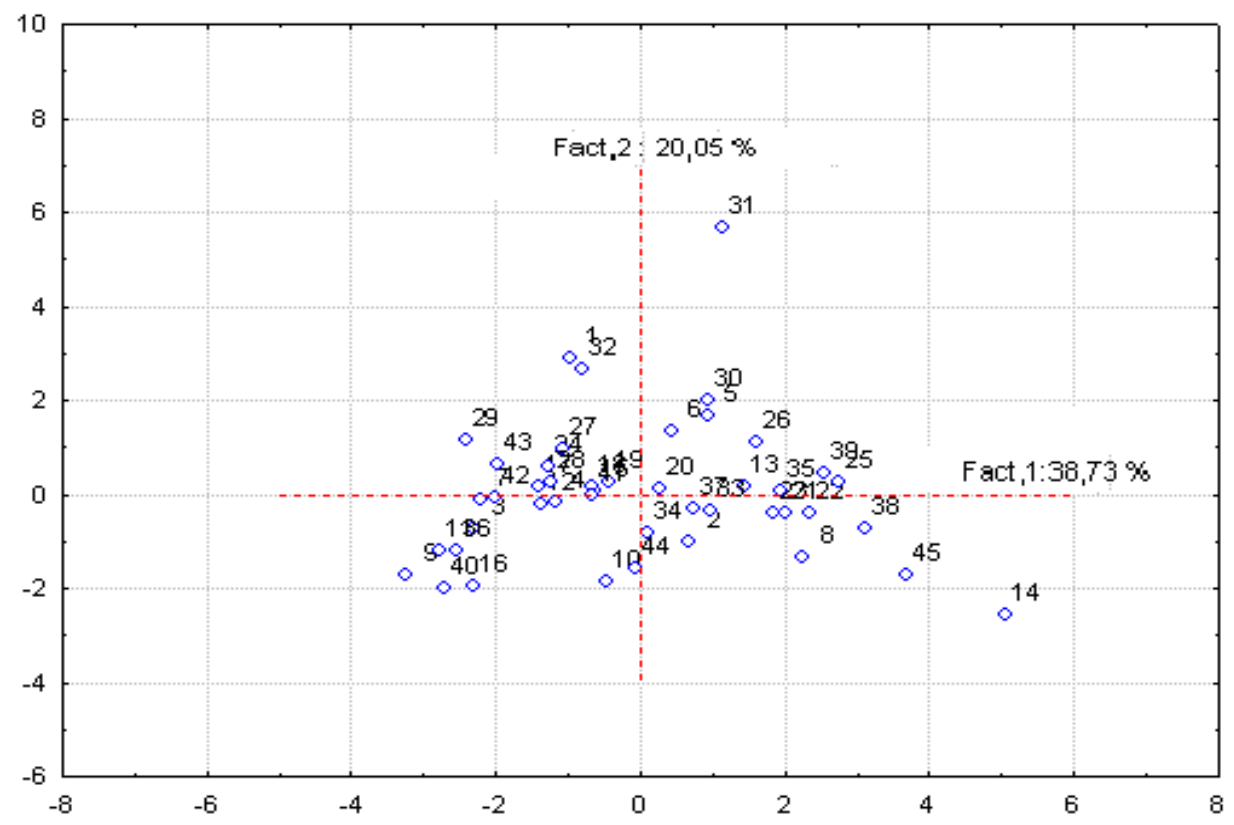

Figure 5 : Projection des unités statistiques dans l'espace factoriel F1-F2.

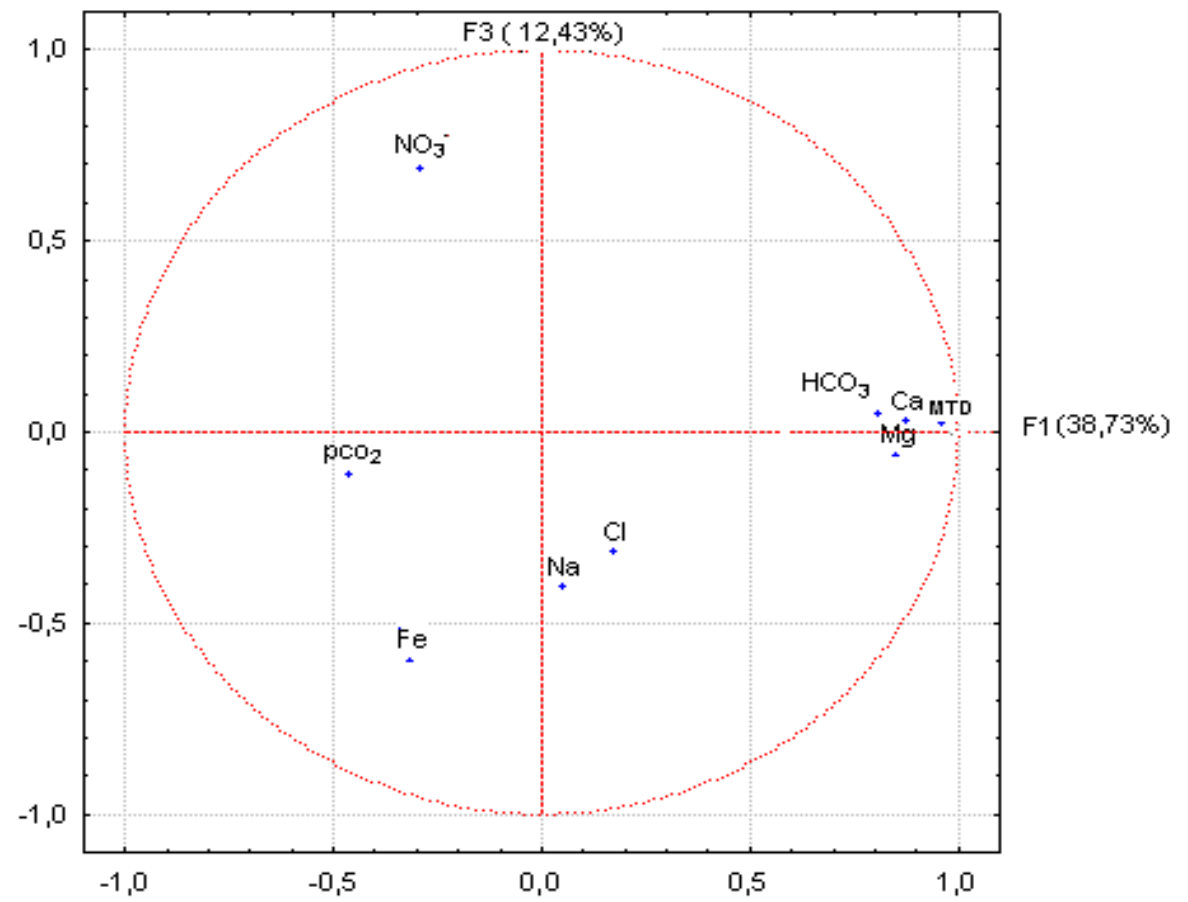

Figure 6 : Projection des variables dans l'espace factoriel F1-F3. 
T. LASM et al. / Int. J. Biol. Chem. Sci. 5(2): 642-662, 2011

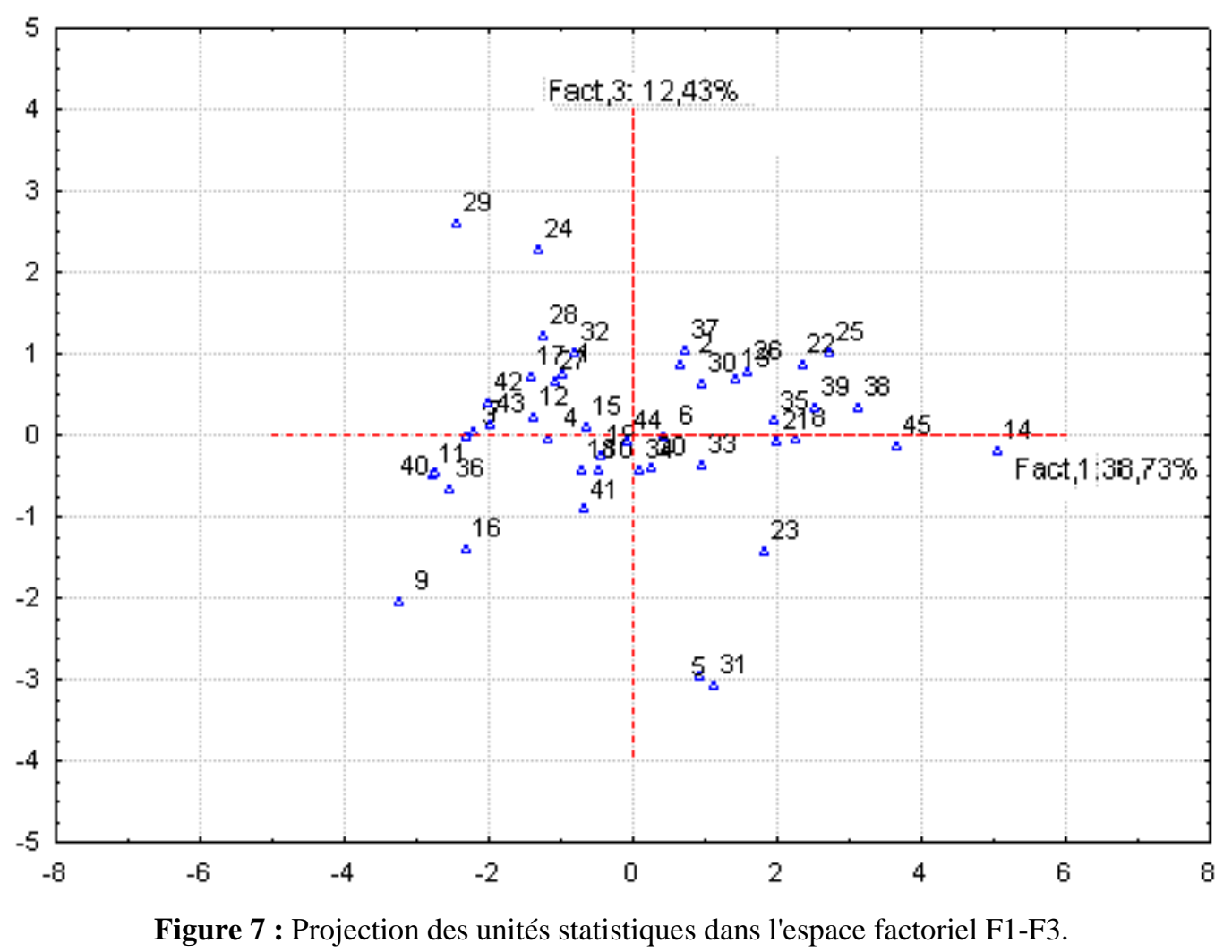


T. LASM et al. / Int. J. Biol. Chem. Sci. 5(2): 642-662, 2011

Tableau 1 : Paramètres mesurés dans les eaux souterraines.

\begin{tabular}{|c|c|c|c|c|c|c|c|c|c|c|c|c|c|c|c|c|c|c|c|c|c|}
\hline Nom & $\mathbf{N}^{\circ}$ & $\mathbf{T}^{\circ} \mathbf{C}$ & pH & CE & $\mathrm{Ca}^{2+}$ & $\mathrm{Mg}^{2+}$ & $\mathrm{Na}^{+}$ & $\mathbf{K}^{+}$ & $\mathrm{Fe}^{2+}$ & $\mathrm{HCO}_{3}$ & Cl- & $\mathrm{SO}_{4}^{2-}$ & $\mathrm{NO}_{2}^{-}$ & $\mathrm{NO}_{3}{ }^{-}$ & THT & $\mathrm{pCO}_{2}$ & MTD & $\mathrm{CO}_{2} \mathrm{D}$ & ISC & ISD & pHéq \\
\hline & & ${ }^{\circ} \mathrm{C}$ & & $\mu \mathrm{SS} . \mathrm{cm}^{-1}$ & $\mathrm{mg} \cdot \mathrm{L}^{-1}$ & $\mathrm{mg} . \mathrm{L}^{-1}$ & $\mathrm{mg} \cdot \mathrm{L}^{-1}$ & $\underset{1}{\mathrm{mg} . \mathrm{L}}$ & $\mathrm{mg} \cdot \mathrm{L}^{-1}$ & $\mathrm{mg} \cdot \mathrm{L}^{-1}$ & $\mathrm{mg} \cdot \mathrm{L}^{-1}$ & $\mathrm{mg} \cdot \mathrm{L}^{-1}$ & $\mathrm{mg} \cdot \mathrm{L}^{-1}$ & $\mathrm{mg} \cdot \mathrm{L}^{-1}$ & ${ }^{\circ} \mathrm{F}$ & atm & $\mathrm{mg} \cdot \mathrm{L}^{-1}$ & $\mathrm{mg} \cdot \mathrm{L}^{-1}$ & & & \\
\hline Dagadji & 1 & 29 & 6,6 & 265,00 & 02,70 & 5,06 & 57,13 & 3,1 & 1 & 49,00 & 19,17 & 27,55 & 0,005 & 34,92 & 3,00 & 0,01 & 199,64 & 18,06 & $-02,59$ & $-05,05$ & 09,19 \\
\hline SCAF & 2 & 29.5 & 7,1 & 38,00 & 10,90 & 6,65 & 9,65 & 33,15 & 0,3 & 384,30 & 09,23 & 04,20 & 0,005 & 9,50 & 5,00 & 0,03 & 467,89 & 44,07 & $-00,61$ & $-01,56$ & 07,71 \\
\hline Do-Sakassou & 3 & 28.5 & 6,3 & 36,00 & 07,60 & 04,60 & 11,30 & 29,25 & 00,60 & 87,00 & 08,87 & 00,60 & 00,01 & 02,12 & 04,00 & 0,05 & 151,95 & 64,95 & $-02,18$ & $-04,72$ & 08,48 \\
\hline Aero kssikro & 4 & 25 & 6,55 & 45,00 & 10,05 & 07,65 & 12,60 & 57,90 & 00,95 & 109,00 & 13,66 & 01,60 & 00,01 & 03,52 & 06,00 & 0,03 & 216,94 & 47,06 & $-01,78$ & $-03,88$ & 08,33 \\
\hline Zilekro & 5 & 25 & 6,8 & 102,00 & 28,70 & 08,80 & 45,05 & 78,30 & 00,85 & 154,00 & 67,10 & 04,20 & 00,05 & 00,40 & 11,00 & 0,02 & 387,45 & 36,64 & $-00,97$ & $-02,65$ & 07,77 \\
\hline Blaisekro & 6 & 25 & 7,1 & 60,20 & 11,41 & 11,62 & 16,25 & 40,35 & 00,25 & 118,37 & 28,40 & 02,60 & 00,01 & 00,98 & 08,00 & 0,01 & 230,24 & 14,35 & $-01,15$ & $-02,49$ & 08,25 \\
\hline Broukro & 8 & 25 & 7,1 & 111,90 & 52,50 & 21,60 & 14,20 & 60,90 & 00,70 & 296,55 & 14,20 & 16,00 & 00,01 & 00,45 & 22,00 & 0,02 & 477,11 & 35,01 & $-00,14$ & $-00,87$ & 07,24 \\
\hline Yaokro & 9 & 25 & 6,3 & 204,00 & 05,10 & 03,30 & 23,08 & 01,60 & 03,70 & 93,00 & 09,23 & 24,02 & 00,01 & 00,90 & 03,00 & 0,05 & 163,94 & 71,79 & $-02,38$ & $-05,15$ & 08,68 \\
\hline Daro & 10 & 25 & 6,8 & 313,00 & 19,30 & 18,05 & 01,86 & 01,90 & 02,35 & 187,00 & 06,03 & 24,72 & 00,01 & 00,78 & 12,00 & 0,03 & 262,00 & 44,94 & $-01,03$ & $-02,30$ & 07,83 \\
\hline Kpakobo & 11 & 25 & 6,3 & 146,30 & 05,40 & 03,62 & 09,06 & 02,45 & 01,60 & 89,00 & 07,81 & 21,90 & 00,01 & 01,74 & 03,00 & 0,05 & 142,59 & 69,04 & $-02,37$ & $-05,10$ & 08,67 \\
\hline Jeanokro & 12 & 25 & 6,55 & 39,30 & 09,90 & 05,55 & 13,00 & 44,25 & 00,81 & 107,39 & 06,39 & 00,45 & 00,01 & 00,45 & 05,00 & 0,03 & 188,20 & 46,58 & $-01,78$ & $-04,02$ & 08,33 \\
\hline Rogerkro & 13 & 25 & 7,25 & 77,70 & 27,62 & 16,60 & 13,25 & 51,30 & 00,20 & 213,57 & 12,24 & 01,20 & 00,01 & 01,47 & 14,00 & 0,01 & 337,46 & 18,12 & $-00,38$ & $-01,19$ & 07,63 \\
\hline Amadoukro & 14 & 25 & 7,05 & 220,00 & 142,30 & 34,35 & 18,50 & 44,29 & 00,60 & 260,14 & 11,71 & 229,40 & 00,01 & 00,45 & 50,00 & 0,02 & 741,75 & 33,34 & 00,11 & $-00,59$ & 06,94 \\
\hline Doba F1 & 15 & 25 & 6,7 & 256,00 & 17,00 & 08,90 & 31,36 & 01,80 & 00,60 & 138,00 & 06,03 & 38,83 & 00,01 & 03,24 & 08,00 & 0,03 & 245,77 & 41,86 & $-01,32$ & $-03,11$ & 08,02 \\
\hline Doba F3 & 17 & 25 & 6,4 & 239,00 & 12,40 & 08,80 & 16,66 & 01,80 & 00,80 & 79,00 & 09,94 & 44,47 & 00,01 & 15,96 & 07,00 & 0,03 & 189,84 & 48,08 & $-01,98$ & $-04,31$ & 08,38 \\
\hline Petit-Pedro & 18 & 25 & 6,65 & 62,00 & 22,06 & 05,21 & 20,05 & 44,40 & 00,60 & 134,20 & 20,76 & 03,20 & 00,01 & 00,60 & 08,00 & 0,03 & 251,09 & 45,79 & $-01,26$ & $-03,34$ & 07,91 \\
\hline Goussikro F1. & 19 & 25 & 6,7 & 61,80 & 11,90 & 11,21 & 17,20 & 25,95 & 00,30 & 130,58 & 22,72 & 01,60 & 00,01 & 00,52 & 08,00 & 0,03 & 221,99 & 39,82 & $-01,48$ & $-03,19$ & 08,18 \\
\hline Goussikro F2 & 20 & 25 & 6,8 & 78,50 & 25,50 & 12,08 & 18,20 & 22,50 & 00,10 & 168,41 & 27,33 & 15,00 & 00,01 & 00,45 & 11,00 & 0,03 & 289,58 & 40,42 & $-00,96$ & $-02,45$ & 07,76 \\
\hline Kablake & 21 & 25 & 7,15 & 106,30 & 51,05 & 17,35 & 21,10 & 52,80 & 00,30 & 257,50 & 19,88 & 07,00 & 00,01 & 01,47 & 20,00 & 0,02 & 428,46 & 27,20 & $-00,16$ & $-00,98$ & 07,31 \\
\hline Bledje & 22 & 25 & 7,35 & 512,00 & 66,00 & 11,90 & 18,00 & 02,50 & 00,25 & 320,00 & 07,81 & 06,39 & 00,01 & 04,20 & 21,00 & 0,01 & 437,06 & 21,34 & 00,25 & $-00,45$ & 07,10 \\
\hline
\end{tabular}


T. LASM et al. / Int. J. Biol. Chem. Sci. 5(2): 642-662, 2011

\begin{tabular}{|c|c|c|c|c|c|c|c|c|c|c|c|c|c|c|c|c|c|c|c|c|c|}
\hline Kandakro & 23 & 25 & 7 & 667,00 & 32,20 & 15,40 & 17,80 & 08,50 & 00,20 & 338,00 & 52,54 & 32,48 & 00,01 & 05,28 & 14,00 & 0,03 & 502,41 & 50,45 & $-00,39$ & $-01,30$ & 07,39 \\
\hline Taboké & 24 & 25 & 6,2 & 364,00 & 31,90 & 09,00 & 11,80 & 15,50 & 00,05 & 63,00 & 20,60 & 112,00 & 00,08 & 52,88 & 12,00 & 0,04 & 316,77 & 59,72 & $-01,91$ & $-04,57$ & 08,11 \\
\hline Benjaminkro & 25 & 25 & 8,05 & 533,00 & 48,50 & 14,90 & 14,00 & 01,82 & 00,20 & 317,00 & 13,50 & 16,26 & 00,01 & 04,80 & 18,00 & 0,00 & 430,99 & 4,23 & 00,82 & 00,92 & 07,23 \\
\hline Hannie & 26 & 25 & 7,8 & 429,00 & 17,60 & 12,90 & 20,75 & 02,40 & 00,22 & 208,00 & 14,55 & 93,13 & 00,01 & 03,84 & 10,00 & 0,00 & 373,40 & 4,95 & $-00,05$ & $-00,43$ & 07,85 \\
\hline Nane & 28 & 25 & 6,6 & 200,00 & 15,70 & 06,00 & 06,90 & 01,46 & 00,13 & 105,00 & 07,45 & 00,45 & 00,01 & 10,44 & 06,00 & 0,03 & 153,54 & 40,70 & $-01,54$ & $-03,69$ & 08,14 \\
\hline Djihimbo & 29 & 25 & 6,2 & 117,70 & 03,80 & 02,20 & 06,10 & 00,90 & 00,27 & 44,00 & 06,40 & 00,45 & 00,01 & 39,30 & 02,00 & 0,03 & 103,43 & 43,44 & $-02,90$ & $-06,24$ & 09,10 \\
\hline Henrikro & 30 & 25 & 7,6 & 517,00 & 40,00 & 13,20 & 36,00 & 01,40 & 00,18 & 02,56 & 11,00 & 151,00 & 00,01 & 04,14 & 15,00 & 0,00 & 259,44 & 0,09 & $-01,81$ & $-04,30$ & 09,41 \\
\hline Belem & 31 & 25 & 8 & 599,00 & 39,00 & 02,60 & 147,70 & 05,20 & 00,12 & 03,23 & 46,15 & 00,45 & 00,01 & 04,26 & 11,00 & 0,00 & 248,72 & 0,05 & $-01,31$ & $-03,99$ & 09,31 \\
\hline Belem 2 & 32 & 25 & 7 & 279,00 & 11,30 & 02,40 & 28,90 & 03,06 & 00,35 & 01,68 & 08,87 & 00,45 & 00,02 & 05,82 & 04,00 & 0,00 & 62,85 & 0,26 & $-03,05$ & $-06,98$ & 10,05 \\
\hline Djapadji 1 & 33 & 25 & 7,1 & 362,00 & 28,55 & 19,95 & 40,51 & 02,10 & 01,50 & 212,00 & 05,32 & 22,61 & 00,01 & 03,42 & 15,00 & 0,02 & 335,97 & 25,30 & $-00,53$ & $-01,42$ & 07,63 \\
\hline Djapadji 2 & 34 & 25 & 6,9 & 340,00 & 25,30 & 15,54 & 30,43 & 01,70 & 01,55 & 200,00 & 06,32 & 26,84 & 00,01 & 03,30 & 13,00 & 0,03 & 310,99 & 37,98 & $-00,80$ & $-02,01$ & 07,70 \\
\hline Djapadji 3 & 35 & 25 & 7,2 & 462,00 & 54,50 & 12,80 & 37,06 & 01,30 & 00,70 & 223,00 & 06,03 & 139,70 & 00,01 & 02,58 & 19,00 & 0,01 & 477,65 & 20,83 & $-00,16$ & $-01,15$ & 07,36 \\
\hline Djapadji 4 & 36 & 25 & 6,3 & 133,40 & 07,60 & 06,77 & 05,09 & 01,85 & 02,55 & 70,00 & 05,68 & 21,90 & 00,02 & 01,14 & 05,00 & 0,04 & 122,60 & 54,21 & $-02,32$ & $-04,90$ & 08,62 \\
\hline Adamadougou & 37 & 25 & 7 & 83,00 & 57,05 & 05,08 & 10,70 & 28,05 & 00,20 & 224,55 & 05,32 & 04,60 & 00,01 & 05,12 & 16,00 & 0,02 & 340,68 & 33,82 & $-00,30$ & $-01,85$ & 07,30 \\
\hline Kuassidrokro & 38 & 25 & 7,4 & 110,40 & 52,36 & 24,50 & 16,00 & 54,75 & 00,26 & 316,08 & 15,62 & 05,20 & 00,01 & 00,45 & 23,00 & 0,01 & 485,23 & 18,69 & 00,18 & $-00,16$ & 07,22 \\
\hline Lamenee & 39 & 25 & 7,65 & 107,80 & 47,80 & 12,50 & 43,20 & 30,30 & 00,15 & 317,30 & 09,58 & 12,80 & 00,01 & 03,06 & 17,00 & 0,01 & 476,70 & 10,60 & 00,40 & 00,03 & 07,25 \\
\hline Koffikro 2 & 41 & 25 & 6,6 & 267,00 & 14,10 & 11,70 & 38,31 & 02,60 & 01,30 & 124,00 & 14,91 & 49,41 & 00,01 & 02,16 & 08,00 & 0,03 & 258,50 & 47,19 & $-01,55$ & $-03,38$ & 08,15 \\
\hline Golykuassikro & 42 & 25 & 6,3 & 31,00 & 07,19 & 03,50 & 11,80 & 25,20 & 00,15 & 70,78 & 07,81 & 03,20 & 00,01 & 00,45 & 03,00 & 0,04 & 130,09 & 55,04 & $-02,33$ & $-05,18$ & 08,63 \\
\hline Dagadji 2 & 43 & 25 & 6,3 & 144,50 & 03,30 & 04,56 & 22,64 & 03,20 & 01,30 & 49,00 & 11,00 & 20,50 & 00,01 & 10,56 & 03,00 & 0,03 & 126,07 & 38,01 & $-02,84$ & $-05,74$ & 09,14 \\
\hline Petit Bouaké & 44 & 25 & 6,45 & 39,00 & 52,50 & 21,60 & 14,20 & 36,00 & 00,80 & 106,17 & 05,85 & 00,45 & 00,03 & 00,67 & 22,00 & 0,04 & 238,27 & 56,65 & $-01,21$ & $-03,01$ & 07,66 \\
\hline Dozo-Djile & 45 & 25 & 7,15 & 169,90 & 103,90 & 23,50 & 14,10 & 67,05 & 00,70 & 263,60 & 15,08 & 157,60 & 00,02 & 00,45 & 36,00 & 0,02 & 646,00 & 27,17 & 00,11 & $-00,63$ & 07,04 \\
\hline
\end{tabular}


Tableau 2 : Matrice de corrélation.

\begin{tabular}{cccccccccc}
\hline & $\mathbf{C a}^{2+}$ & $\mathbf{M g}^{2+}$ & $\mathbf{N a}^{+}$ & $\mathbf{F e}^{2+}$ & $\mathbf{H C O}_{3}^{-}$ & $\mathbf{C l}^{-}$ & $\mathbf{N O}_{3}^{-}$ & $\mathbf{p C O}$ & $\mathbf{M T D}$ \\
\hline $\mathbf{C a}^{2+}$ & 1,00 & & & & & & & & \\
$\mathbf{M g}^{2+}$ & 0,77 & 1,00 & & & & & & & \\
$\mathbf{N a}^{+}$ & 0,07 & $-0,11$ & 1,00 & & & & & & \\
$\mathbf{F e}^{2+}$ & $-0,23$ & $-0,04$ & $-0,07$ & 1,00 & & & & & \\
$\mathbf{H C O}_{3}^{-}$ & 0,54 & 0,61 & $-0,21$ & $-0,18$ & 1,00 & & & & \\
$\mathbf{C l}^{-}$ & 0,03 & 0,00 & 0,45 & $-0,22$ & 0,04 & 1,00 & & & \\
$\mathbf{N O}_{3}^{-}$ & $-0,18$ & $-0,25$ & 0,01 & $-0,16$ & $-0,28$ & $-0,01$ & 1,00 & & \\
$\mathbf{p C O}_{2}$ & $-0,31$ & $-0,27$ & $-0,41$ & 0,39 & $-0,20$ & $-0,15$ & 0,03 & 1,00 & \\
$\mathbf{M T D}$ & 0,85 & 0,78 & 0,04 & $-0,24$ & 0,83 & 0,20 & $-0,17$ & $-0,32$ & 1,00 \\
\hline
\end{tabular}


Le regroupement des points d'eaux des villages Amadoukro et Dozo-djilé à l'extrémité positive de l'axe $\mathrm{F} 1$ et la localisation du forage Belem à l'extrémité positive de l'axe F2 dans l'espace des unités statistiques (Figure 5) est en accord avec la position des points dans l'espace des variables. Il révèle que la minéralisation des eaux à Amadoukro (Ech. 14) et Dozo-Djilé (Ech. 45) est caractérisée par les fortes teneurs en calcium, magnésium et bicarbonates par contre l'eau du forage Belem F1 (Ech. 31) est marquée par une abondance en chlorures et en sodium.

La plupart des points d'eau échantillonnés, soit 93\%, se regroupent à l'intersection des axes F1 et F2, traduisant la faible minéralisation des eaux de forages de la région.

La projection des variables dans l'espace factoriel F1-F3 (Figure 6) confirme que le facteur F1 est celui qui exprime la minéralisation liée au temps de séjour des eaux souterraines de la région. Le facteur F3 est déterminé par le nitrate $\left(\mathrm{NO}_{3}{ }^{-}\right)$et le fer $\left(\mathrm{Fe}^{2+}\right)$, deux variables situées à des positions diamétralement opposées sur l'axe F3. Ces variables étaient mal définis dans le plan factoriel F1-F2, ce n'est donc qu'au niveau du plan factoriel F1-F3 qu'elles sont bien exprimées. La présence des nitrates sur l'axe F3 indique que cet axe est gouverné par les phénomènes d'apports superficiels. En effet, la présence des nitrates dans les eaux souterraines est indicatrice d'un apport superficiel provenant des activités anthropiques (déforestation, usage des engrais, élevage industriel, etc.). Les nitrates parviennent dans les eaux souterraines à partir des eaux de pluies qui s'infiltrent dans le sol. L'opposition du fer aux nitrates sur l'axe F3 indique que cette variable a une origine différente de celle des nitrates. En effet, le fer présent dans les eaux souterraines de SanPedro résulterait de l'hydrolyse acide des minéraux ferromagnésiens (pyroxènes, amphiboles, biotites, etc.) rencontrés dans les roches cristallines et métamorphiques de cette région. Le facteur 3 exprime donc l'hydrolyse acide des minéraux.

La répartition des échantillons dans le plan factoriel F1-F3 (Figure 7) confirme les interprétations faites sur les eaux de forages d'Amadoukro et Dozo-Djilé. En outre, elle révèle la forte proportion du nitrate $(52,88$ mg. $\mathrm{L}^{-1}$ ) dans l'eau de forage de Taboké, village situé non loin des grandes plantations d'hévéaculture. L'analyse comparative de l'espace factoriel F1-F3 des unités statistiques avec celui des variables permet de confirmer la teneur élevée du fer à Yaokro $\left(3,7 \mathrm{mg} \cdot \mathrm{L}^{-1}\right)$.

\section{DISCUSSION}

Les teneurs moyennes des ions contenus dans les eaux souterraines de San-Pedro s'inscrivent dans les normes de potabilité des eaux pour la consommation humaine d'une manière générale. Les teneurs en nitrates $\left(\mathrm{NO}_{3}{ }^{-}\right)$et en fer $\left(\mathrm{Fe}^{2+}\right)$ respectivement dans les forages Taboké $\left(52,9 \mathrm{mg} . \mathrm{L}^{-1}\right)$ et Yaokro $(3,7$ $\left.\mathrm{mg} . \mathrm{L}^{-1}\right)$ sont légèrement au dessus des normes recommandées par l'Organisation Mondiale de la Santé (OMS). Les eaux de deux forages $(4,4 \%)$ présentent des duretés indésirables ce qui les rend impropres à la consommation humaine. Il s'agit des forages de Dozo-djilé $\left(36{ }^{\circ} \mathrm{F}\right)$ et d'Amadoukro $\left(50{ }^{\circ} \mathrm{F}\right)$. Des observations analogues ont été aussi faites dans d'autres régions du pays, notamment dans la région de Tiassalé (Sud de la Côte d'Ivoire) où les travaux de Lasm et al. (2008b) ont mis en évidence une proportion importante de forages $(22 \%)$ à forte dureté. Cette différence pourrait s'expliquer d'une part par l'intensité des réactions hydrogéochimiques et d'autre part par le contexte pétrographique de ces régions. L'acidité des eaux est principalement liée à la production de $\mathrm{CO}_{2}$ dans les couches superficielles du sol. Cette interprétation est en accord avec la présence d'une zone forestière dans la région de San-Pedro. Les 
faibles valeurs $\mathrm{du} \mathrm{pH}$ sont donc la conséquence de la forte pression du $\mathrm{CO}_{2}$ en équilibre avec les eaux souterraines comme ceci a été déjà démontré dans les régions du Grand Abidjan et du Sud-est de la Côte d'Ivoire (Oga, 1998; Oga et al., 2008 ; Adiaffi et al., 2009). En effet, les eaux de précipitation en s'infiltrant pour atteindre les aquifères souterrains profonds vont s'acidifier par le $\mathrm{CO}_{2}$ dissous produit par les sols sous couvert végétal dense. Les teneurs élevées de la $\mathrm{pCO}_{2}$ dans les eaux de forages suggèrent que les eaux souterraines sont issues d'un système ouvert sur le $\mathrm{CO}_{2}$ du sol (Njitchoua, 1997 in Soro 2002). En effet, le $\mathrm{CO}_{2}$ équilibrant avec l'eau souterraine est celui produit dans la zone non saturée du sol, au terme de la décomposition de la matière organique. Ces eaux souterraines ont une température homogène d'une manière générale avec une température moyenne de 25,6 ${ }^{\circ} \mathrm{C}$. Les températures mesurées sont en accord avec la température annuelle de l'air ambiant de la région au cours de ces dernières décennies compris entre 25 et $30^{\circ} \mathrm{C}$.

Les eaux souterraines du Sud-Ouest de la Côte d'Ivoire sont faiblement minéralisées à l'instar des eaux souterraines des régions de Grand Lahou (Sud-ouest), Abengourou (Est), et Katiola (Centre-nord). Cette faible minéralisation des eaux est conforme aux observations de Sarrot et al. (1992) dans les eaux souterraines du socle cristallin qui indiquent une moyenne autour de $120 \mathrm{mg} . \mathrm{L}^{-1}$. Les matières totales dissoutes des eaux souterraines (MTD) dépendent de la pétrographie de la formation rocheuse et du temps de résidence de l'eau dans l'aquifère (Adiaffi et al., 2009). Ces auteurs ont montré qu'il existe une bonne relation entre la minéralisation totale des eaux souterraines (MTD) et le temps de résidence moyen de l'eau souterraine. Dans la région d'Abidjan (Sud de la Côte d'Ivoire), Adiaffi et al. (2009) montrent que pour un temps de résidence moyen des eaux d'environ 10000 ans, l'hydrolyse des minéraux silicatés conduit éventuellement au niveau de l'aquifère du socle sous couvert des dépôts du Continental Terminal (CT), à une eau saturée vis-à-vis de la calcite. L'aquifère n'étant pas carbonaté, le calcium présent dans les eaux provient des minéraux primaires calciques. Dans les aquifères de socle fissuré où la production des bicarbonates $\left(\mathrm{HCO}_{3}{ }^{-}\right)$est due principalement de l'altération de minéraux silicatés, sa teneur renseigne sur le temps de séjour de l'eau dans l'aquifère.

Les eaux souterraines de la région de San-Pedro sont majoritairement (80\%) bicarbonatées comme la plupart des eaux souterraines en région de socle d'Afrique de l'Ouest (Oga, 1998; Soro, 2002; Diop et Tijani, 2008 ; Adiaffi, 2008 ; Adiaffi et al., 2009). Dans la région du Grand Abidjan, les travaux d'Oga (1998) ont montré pour les eaux du socle un faciès bicarbonaté avec deux tendances, calcique et magnésienne. Dans la région de Grand-Lahou, située à l'Est de la zone d'investigation, Soro (2002) a obtenu des résultats similaires. La prédominance des ions $\mathrm{HCO}_{3}{ }^{-}$et $\mathrm{Cl}^{-}$est une caractéristique des eaux de socle de Côte d'Ivoire (Soro, 2002).

Différents phénomènes interviennent dans l'acquisition de la minéralisation des eaux souterraines du Sud-Ouest de la Côte d'Ivoire. En effet, la minéralisation des eaux souterraines dépend du facteur temps de séjour et de l'interaction eau-roche dans les aquifères, permettant la mise en solution des ions bicarbonates, calcium et magnésium après une hydrolyse acide des plagioclases. Les ions nitrates, sodium et chlorures contenus dans les eaux souterraines proviennent des horizons superficiels lessivés par les eaux d'infiltration. Les travaux menés par Lasm et al. (2008b) dans la région de Tiassalé, au sud de la Côte d'Ivoire ont conduit à des résultats similaires. Cependant, le processus d'acquisition du fer dans les eaux souterraines reste différent. En effet, le fer provient du lessivage des horizons superficiels 
et de l'hydrolyse acide des minéraux ferromagnésiens des roches encaissantes respectivement dans les régions de Tiassalé et de San-Pedro. La présence du fer dans les eaux souterraines pourrait aussi résulter d'un processus de dénitrification de la pyrite et de la chalcopyrite (Martelat et al., 1997). L'absence de ces minéraux dans le cortège pétrographique et métallogénique de cette région ne permet pas de retenir cette interprétation. Le sous-sol de cette région est constitué de roches cristallines et métamorphiques dont l'altération favorise la dissolution des minéraux ferromagnésiens (biotites, pyroxènes, amphiboles, etc.) et leur transport vers les eaux souterraines. L'interprétation de l'origine du fer dans les eaux souterraines de San-Pedro est donc en accord avec la géologie de cette région.

La sous saturation des eaux étudiées vis-à-vis des carbonates est une caractéristique des eaux souterraines du socle fissuré de l'Afrique de l'Ouest en général et de la Côte d'Ivoire en particulier (Oga, 1998 ; Soro, 2002 ; Adiaffi, 2008 ; Adiaffi et al., 2009). En effet, l'absence de roches carbonatées dans le cortège pétrographique de la Côte d'Ivoire explique parfaitement ce résultat.

Les travaux de Cathelineau et al. (1999) ont montré que les sels $\left(\mathrm{Na}^{+}\right.$et $\left.\mathrm{Cl}^{-}\right)$présents dans les fluides des fractures profondes des granites de la Vienne (France) proviennent de l'altération probable des minéraux primaires des roches contenant $\mathrm{Na}^{+}$et $\mathrm{Cl}^{-}$ou de la dissolution d'évaporites préalablement introduites dans la roche lors des paléocirculations au Mésozoïque. Les sels libérés (sodium et chlorures) vont être transportés vers les eaux souterraines. Dans le cas du SudOuest de la Côte, la présence d'évaporites n'a été évoquée dans aucune étude géologique. Cette hypothèse serait difficilement applicable dans la région d'étude. La forte proportion du nitrate $\left(52,88 \mathrm{mg} . \mathrm{L}^{-1}\right)$ dans l'eau de forage de Taboké, indique une probable contamination, très localisée, due à l'utilisation des engrais dans les grandes plantations industrielles d'hévéaculture qui s'y trouvent.

\section{Conclusion}

$\mathrm{Au}$ terme de cette étude, plusieurs résultats importants ont été obtenus sur les aquifères fissurés de cette région. Les eaux souterraines de la région de San-Pedro sont faiblement minéralisées, froides et douces. Ces eaux sont caractérisées par une dureté convenable à la consommation humaine et par une alcalinité bicarbonatée. Les eaux sont réparties en quatre hydrofaciès : les eaux bicarbonatées calciques et magnésiennes (60\%), les eaux bicarbonatées sodiques et potassiques $(20 \%)$, les eaux chlorurées sulfatées calciques et magnésiennes $(11 \%)$ et les eaux chlorurées sodiques et potassiques (9\%). Les valeurs des ISC et ISD indiquent d'une manière générale que les eaux de la région de San-Pedro sont sous saturées vis-àvis de la calcite et de la dolomite. Ceci est en accord avec l'absence de roches carbonatées dans cette région. En fonction de leur vitesse de circulation, les eaux souterraines ont été subdivisées en trois familles d'eau d'âges relatifs : les eaux à circulation très lente $(13 \%)$, les eaux à circulation lente $(56 \%)$ et les eaux à circulation rapide $(31 \%)$. Les différents phénomènes d'acquisition de la minéralisation des eaux souterraines du SudOuest de la Côte d'Ivoire sont le temps de séjour des eaux dans les aquifères, l'hydrolyse acide des roches encaissantes et l'infiltration des eaux de pluies contenant des embruns marins.

En perspective, nous comptons entreprendre une étude isotopique des eaux souterraines afin de comprendre le mécanisme de recharge des aquifères et déterminer les âges absolus des eaux souterraines de la région de San-Pedro.

\section{REMERCIEMENTS}

Les auteurs remercient les différents instructeurs qui ont contribué à l'amélioration 
de ce présent document à travers leurs critiques, leurs commentaires et suggestions. Les auteurs voudraient aussi remercier les responsables du Ministère des Infrastructures Economiques de la région de San-Pedro (Côte d'Ivoire) qui ont mis à leur disposition les différentes données utilisées dans le cadre de cette étude.

\section{REFERENCES}

Adiaffi B. 2008. Apport de la géochimie isotopique, de l'hydrochimie et de la télédétection à la connaissance des aquifères de la zone de contact "soclebassin sédimentaire" du Sud-Est de la Côte d'Ivoire. Thèse de doctorat, Université Paris-Sud, Orsay, France, p. 217.

Adiaffi B, Marlin C, Oga YMS, Massault M, Noret A, Biémi J. 2009. Palaeoclimatic and deforestation effect on the coastal fresh groundwater resources of SE Ivory Coast from isotopic and chemical evidence. J. Hydrol., 1-2: 130-141.

Cathelineau M, Cuney M, Boiron MC, Coulibaly Y, Ougougdal AM. 1999. Paléopercolations et paléointeractions fluides/roches dans les plutonites de Charroux-Civray. In Etude du Massif de Charroux-Civray. EDP Sciences ; 159179.

Diop S, Tijani MN. 2008. Assessing the basement aquifers of Eastern Senegal. Hydrogeol. Journ., 16: 1349-1369.

Géohive. 2008. Population du Bas-Sassandra. www.tlfq.ulaval.ca/axl/afrique/cotiv.htm, page consultée en Mai 2008.

Géomines Ltée. 1982. Inventaire hydrogéologique appliqué à l'hydraulique villageoise. Ministère des travaux publics et des transports, Direction Centrale de l'Hydraulique, République de Côte d'Ivoire. Carte de Sassandra, Cahier 33, p.24.

Jourda JP. 2005. Méthodologie d'application des techniques de télédétection et des systèmes d'information géographique à l'étude des aquifères fissurés d'Afrique de l'ouest. Concept de l'Hydrotechnique spatiale : cas des zones tests de la Côte d'Ivoire. Thèse de doctorat ès Sciences Naturelles, Université de CocodyAbidjan, Côte d'Ivoire, p. 429.

Kouamelan AN, Peucat JJ, Delor C. 1997. Reliques archéennes $(3,5 \mathrm{Ga})$ au sein du magmatisme birimien de la Côte d'Ivoire, craton Ouest-Africain. C. R. Acad. Sci., 324: 719-727.

Lasm T. 2000. Hydrogéologie des réservoirs fracturés de socle: Analyse statistique de la fracturation et des propriétés hydrodynamiques. Application à la région des montagnes de Côte d'Ivoire (domaine archéen). Thèse de doctorat, Université de Poitiers, France, p. 274.

Lasm T, Razack M. 2001. Lois d'échelle dans la fracturation des roches dures cristallines et dans le réseau hydrographique associé. C. R. Acad. Sci., 333: 225-232.

Lasm T, Razack M, Youan Ta M. 2008a. Geostistical analysis of the transmissivity in fissured reservoirs of Bondoukou Region (northeast, Côte d'Ivoire). In IAH-Selected Papers book series titled : Applied Groundwater Studies in Africa, Segun Adelana, Alan MacDonald, Tamiru Alemayehu, Callist Tindimugaya (eds). Publishers Taylor \& Francis: The Netherlands; 473-485.

Lasm T, Yao TK. Oga MS, Kouamé F, Jourda P, Kouadio E, Baka D. 2008b. Analyse des caractéristiques physico-chimiques des eaux souterraines en zone de socle protérozoïque de la région de Tiassalé (sud de la Côte d'Ivoire). Euro. J. Sci. Res., 20(3): 526-543.

Martelat A, Foucher JC, Lachassagne P, Pauwels H. 1997. Processus de dénitrification au sein d'un aquifère de socle (schistes à pyrites) : caractérisation $\mathrm{du}$ fonctionnement hydrogéochimique à 
l'échelle locale, dans le cadre d'un petit bassin versant breton. In Hard Rock Hydrosystems, Proceedings of Rabat Symposium S2. IAHS Publication, 241: 29-35.

Oga YMS. 1998. Ressources en eaux souterraines dans la région du Grand Abidjan (Côte d'Ivoire): Approches hydrochimiques et isotopiques. Thèse de doctorat, Université de Paris XI, Orsay, France, p. 241.

Oga YMS, Marlin C, Dever L, Filly A, Njitchoua R. 2008. Hydrochemical and isotopic characteristics of coastal groundwater near Abidjan (southern Ivory Coast). In IAH-Selected Papers book series titled : Applied Groundwater Studies in Africa, Segun Adelana, Alan MacDonald, Tamiru Alemayehu, Callist
Tindimugaya (eds). Publishers Taylor \& Francis: The Netherlands; 371-389.

Sarrot-Reynauld J, Biémi J, Jourda JP, Soro N. 1992. Origine des eaux souterraines du socle cristallin en Côte d'Ivoire. J. Afri. Earth Sci., 15(3/4) : 303-309.

Soro N. 2002. Hydrochimie et géochimie isotopique des eaux souterraines du degré carré de Grand Lahou et ses environs (Sud-Ouest de la Côte d'Ivoire). Implications hydrologiques et hydrogéologiques. Thèse de doctorat ès Sciences Naturelles, Université de Cocody, Abidjan, Côte d'Ivoire, p. 256.

Yacé I. 2002. Initiation à la Géologie. L'exemple de la Côte d'Ivoire et de l'Afrique de l'Ouest. Edition CEDA: Abidjan-Côte d'Ivoire; 183. 